\title{
Data analysis toolkit for long-term, large-scale experiments
}

\author{
D. P. BENNETT ${ }^{1}$, R. J. CUSS ${ }^{2}$, P. J. VARDON ${ }^{3}$, J. F. HARRINGTON ${ }^{2}$, R. N. PHILP ${ }^{1}$ AND H. R. ThOMAS ${ }^{1}$ \\ ${ }^{1}$ Geoenvironmental Research Centre (GRC), Cardiff School of Engineering, Cardiff University, Queen's \\ Buildings, The Parade, Cardiff, CF24 3AA, UK \\ 2 British Geological Survey/NERC, Kingsley Dunham Centre, Keyworth, Nottingham, NG12 5GG, UK \\ ${ }^{3}$ Geo-Engineering Section, Department of Geoscience and Engineering, Delft University of Technology, PO Box \\ 5048, 2600 GA Delft, The Netherlands
}

\section{[Abstract]}

A new data analysis toolkit is presented, suitable for the analysis of large-scale, long-term datasets and the phenomenon/anomalies they possess. The toolkit aims to expose and quantify scientific information in a number of forms contained within a time-series based dataset in a quantitative and rigorous manner, reducing the subjectivity of observations made, thereby supporting the scientific observer. The features contained within the toolkit include the ability to handle non-uniform datasets, time-series component determination, frequency component determination, feature/event detection and characterisation/parameterisation of local behaviours. An application is presented of a case study dataset arising from the 'Lasgit' experiment.

Keywords: time-series analysis, Lasgit, large-scale experiment, large dataset, non-uniform

\section{Introduction}

The development of the safety case for deep geological repositories has prompted a series of experimental activities ranging from laboratory scale to field scale. A proportion of such experiments have a significant duration owing to the longevity of the problems being studied, e.g. the (re)saturation of clay buffers (SKB 2007, Dixon et al. 2002). The large-scale, long-term nature of these experiments can yield vast datasets that would be impractical to examine entirely by hand.

Time-series analysis and signal processing techniques applied computationally can help to reduce subjectivity in observations made (e.g. Chatfield 1989), therefore supporting the scientific observer. This is achieved by providing a uniform and automated procedure for making observations that, by its nature, performs the task in a quantitative way. This approach improves such observations by turning them into measurements.

Such computational analysis may also be instrumental in uncovering a wealth of information contained within the dataset (e.g. Box and Jenkins 1976, Chatfield 1989), while addressing the practicalities of analysing a large-scale dataset. In addition to any primary observations or data processing, an analysis focusing on smaller scale features can be performed to expose this information and can potentially yield extra value from the dataset. Such an analysis is termed a 'second order' analysis in this work.
As an additional consequence of the longevity of such experimental undertakings, non-uniform datasets often arise, often due to circumstances outside of the experimental control such as hardware malfunction/failure. The probability of experiencing an event causing non-uniformity increases with the length of the experiment (Halpern 1978, O'Connor 1995). Typically automated computational analysis algorithms take uniform input data, and as such can be hampered by the non-uniformities present in such datasets (e.g. Box and Jenkins 1976).

This paper describes the development at the GRC of a series of data analysis tools designed around long-term, large-scale datasets capable of performing time-series and signal processing procedures on non-uniform datasets. These approaches, based on statistics, Fourier analysis and component analysis are described within the paper as a 'data analysis toolkit'. Each data processing and analysis component has been chosen based on its potential to expose useful scientific information and implemented in such a way as to accommodate the input of such a dataset. An initial analysis is then presented of a case study dataset that aims to computationally identify and quantify smaller scale and otherwise difficult to observe behaviours and features.

\section{Case study}

An example of a long-term large-scale experiment with a large non-uniform dataset is the Large Scale 
Gas Injection Test or 'Lasgit', a field-scale experiment located at the Äspö Hard Rock Laboratory (HRL) in Sweden. The project is a fullscale demonstration test based on the KBS-3V highlevel radioactive waste disposal concept (SKB 2006) and is designed to examine the impact of gas (generated primarily by corrosion of metal) in compact bentonite within a deposition hole.

Lasgit has been in continuous operation since February 2005 (Cuss et al. 2010) and has undergone more than 90000 logging cycles (i.e. the recording of a single datum point at all installed sensors at a specific time) leading to an acquired dataset in excess of 14.7 million datum points owing to its highly instrumented nature.

As of the end of 2011 three gas injection tests have been undertaken. Tortuous gas flow paths have been detected and a number of externally caused events have been observed within the dataset (Cuss et al. 2010, Cuss et al. 2011). However a large quantity of uninterpreted information may remain within the dataset making it a candidate for analysis of the kind described in this paper.

The Lasgit dataset possesses a number of phenomena that require consideration during computational analysis. Primarily the logging (time) interval is not consistent across the dataset. This non-uniformity is due in part to increases of sample rates during periods of critical interest such as gas injection phases and also to hardware limitations and unavoidable breakdown causing interruptions and loss of data. Some data streams also occasionally 'spike', characterised by a single datum point, disparate from the consensus of surrounding data and therefore can be considered to not be a representative measurement at that time, or of the process of interest at the observed scale.

\section{Data analysis toolkit}

To address the above problems a toolkit capable of performing a 'second order' analysis (as defined above) on long-term, large-scale datasets with nonuniformities has been developed.

\section{Options regarding reformatting of a dataset}

Computational time-series analysis and algorithmic procedures typically require uniform datasets. To overcome the difficulty associated with the computational analysis of a non-uniform dataset the option exists to reprocess the data into a uniform form. One method of achieving this is by down-sampling the dataset to a uniform time step (i.e. taking the points that correspond to the lowest common sampling rate within the dataset) at the cost of a loss of data resolution.

There may exist however, as exampled in the case study dataset, the situation where there is no global timing grid that a base sample rate can be affixed to, i.e. data logged at hourly intervals that correspond with the top of an hour in one section of the dataset do not necessarily correspond to the top of an hour in other sections of the dataset that are sampled at an hour. In the case study dataset this is primarily due to logging hardware requiring restarts at various points in the experimental history, leading to an arbitrary interval between two logging cycles.

An alternative to this approach is to define an appropriate assigned and arbitrary global sample rate for the dataset and to interpolate the defined points using the original non-uniform data. However a high percentage of the original data could be abandoned in favour of the 'created' data using this method. There is also the risk that detail present in the original dataset will be lost in the created dataset, particularly local maxima and minima if they do no coincide closely with an interpolated point.

Neither of these approaches were considered entirely satisfactory therefore an adaption of relevant algorithms to a non-uniform applicability was undertaken.

\section{Generalisation of algorithms to non-uniform input}

Time-series analysis can be considered as the measurement or exploitation of the fact that proximate datum points in a time-series are interconnected to (or non-independent of) each other (Box and Jenkins 1976). Approaching timeseries analysis with this in mind helps ensure that any modification/generalisation of existing algorithms towards non-uniform application preserve the intent of the original algorithms and the mathematics underpinning them.

Processes that involve moving windows across a time-series, i.e. processes that allow the analyst to specify the scale over which the non-independence 
of points is assumed to occur, can be utilised. These can be implemented by either collating a fixed number of points around the centre of the window or by collating all the points that fall within a fixed time span around the centre of the window.

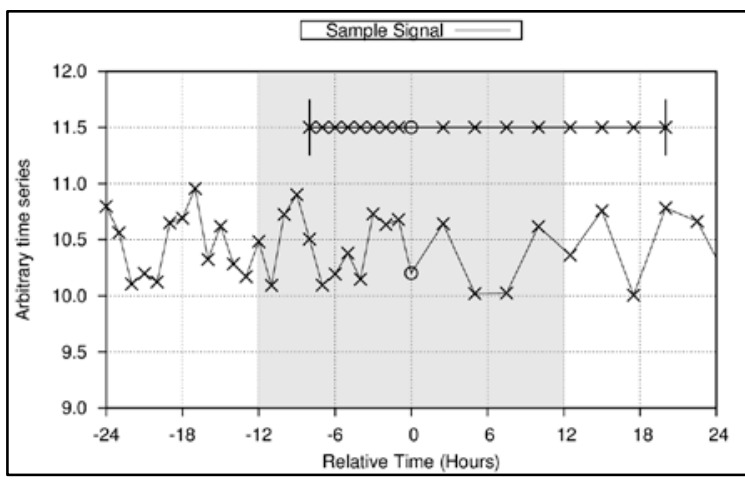

Figure 1 - Comparison of time-series windowing methodologies.

When a time-series has been sampled uniformly the two approaches are equivalent, however when applied to a time-series that is non-uniform, the method using a window fixed in time maintains the scale over which the observation is made. Figure 1 depicts a comparison of the two 'windowing' methods when applied to a time-series at the point the sample rate changes. It can be seen that the approach using a fixed number of points places a forward bias on the actual time indices of the timeseries.

\section{Basic time windowing applications and implications}

Applying time windowing to a weighted moving average algorithm requires a continuous user defined weighting function in place of pre-defined fixed weighting constants used in the fixed number of points windowing method.

This allows the modified algorithm to adapt to non-uniformities in the input time-series by deriving weight as a function of each point's time relative to the centre of the window. Information such as the local mean, standard deviation and characterising values of the window can be calculated.

Spike identification can be achieved by comparing the absolute deviation of a point from its local mean (excluding the point in question) to a threshold defined as a multiple of the local standard deviation around the point (again excluding the point itself). Exceeding that threshold classes the point as a spike, either suggesting rapid process evolution or possible measurement error at that point.

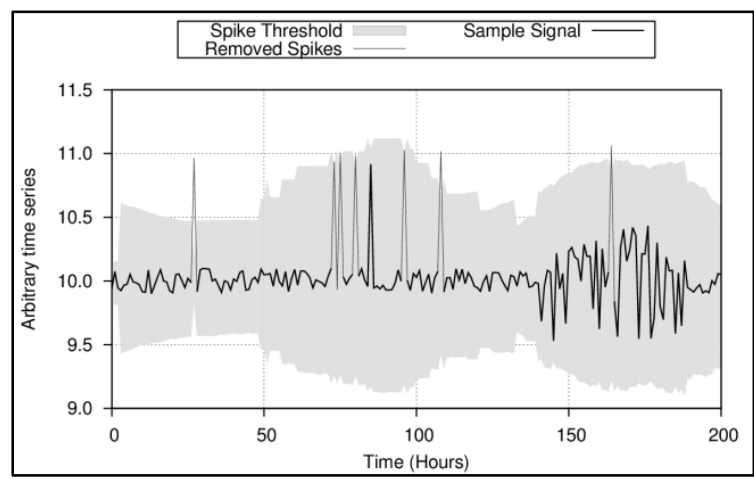

Figure 2 - Evolution of spike threshold with different levels of spike frequency and signal noise.

The time windowing implementation of point gathering to determine the local parameters that are required to make such a comparison ensure that the observed density of spikes with time will be unaffected by a change in sample rate, assuming the frequency or likelihood of spike occurrence is not a function of the sample rate and that the data is sampled at a greater rate than the underlying process evolution of interest.
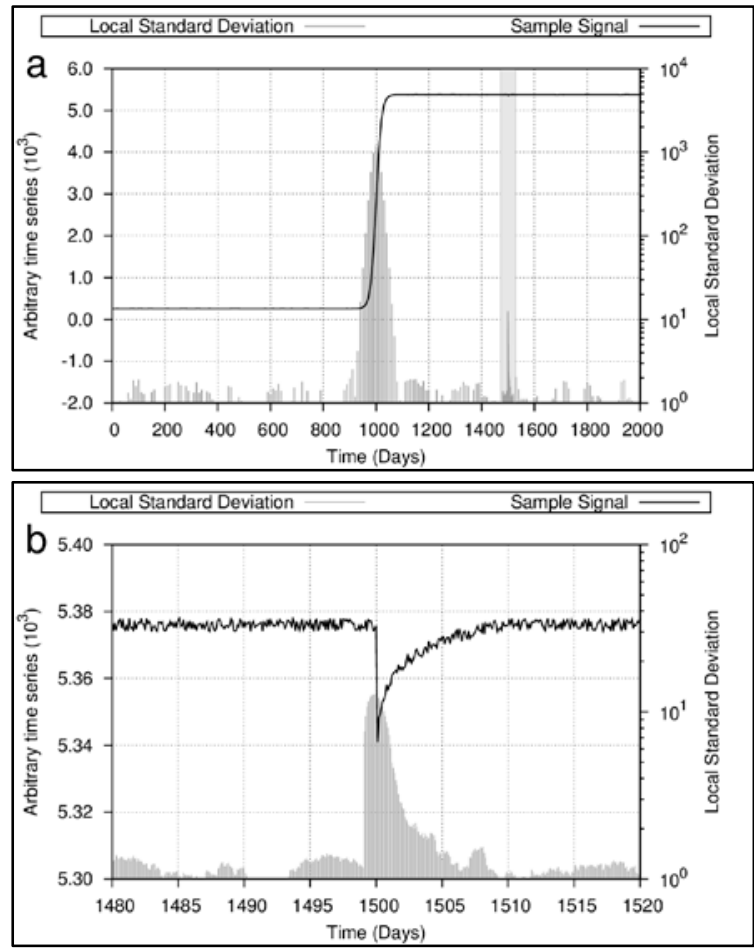

Figure 3 - (a) Local standard deviations of a timeseries. An isolated peak is highlighted as a small scale event candidate. (b) Detailed view of timeseries at section highlighted in (a). 
Comparatively, using a fixed number of points will result in variation in the threshold determination with changes in sample rate. Figure 2 depicts spike threshold evolution along a time-series with varying local environments as determined by time windowing.

\section{Information quantification / parameterisation}

Quantification or parameterisation of local aspects of a dataset with respect to time can potentially expose information about the system it represents. Examples include quantification of local standard deviation of the signal, as discussed in the previous section, to characterise the noise and/or rate of change within the time-series with time. This method uses the time windowing method for point gathering discussed in the previous section.

Isolated peaks in the local standard deviation or changes in the inherent amount of noise within the system can indicate events within the dataset that are candidates for scientific investigation, with their relative prominence suggesting possible relevance. Figure 3 depicts an arbitrary data series, with the local standard deviation with time. The large peak in standard deviation corresponds to the large ramp in time-series magnitude. The local standard deviation peak, highlighted as an 'Anomalous Noise Peak', corresponds to a small scale event in the time-series which is shown in more detail in Figure 3b. The effect identified by the local peak is unlikely to appear significant when observed the macro scale of the dataset as a whole, however, could indicate a second order process occurring.

Aggregation of the derived local standard deviation series across sensor types can be performed allowing a visual indicator of synchronisation with in a group of sensors. The result of this process is shown in Figure 5.

\section{Frequency domain analysis}

Cyclic information over a domain, i.e. frequency components along with their amplitudes and phases, can be quantified with application of a Fourier Transform (FT) analysis. Application to a time-series requires a Discrete Fourier Transform (DFT). The standard DFT process converts a timeseries into a power series consisting of a discrete set of frequency locations defined by the timeseries length and sample rate.
The mathematical representation of a DFT is shown in Equation 1 (Bagchi and Mitra 1999), where $P(k)$ is the power series of the time-series $F$ with points at $n$ or $t_{n}$ (represented by a complex number) at frequencies that are $n$ multiples of $\mathrm{k} / \mathrm{N}$, $\mathrm{N}$ is the number of points in the time-series and $0 \leq \mathrm{k} \leq \mathrm{N}-1$.

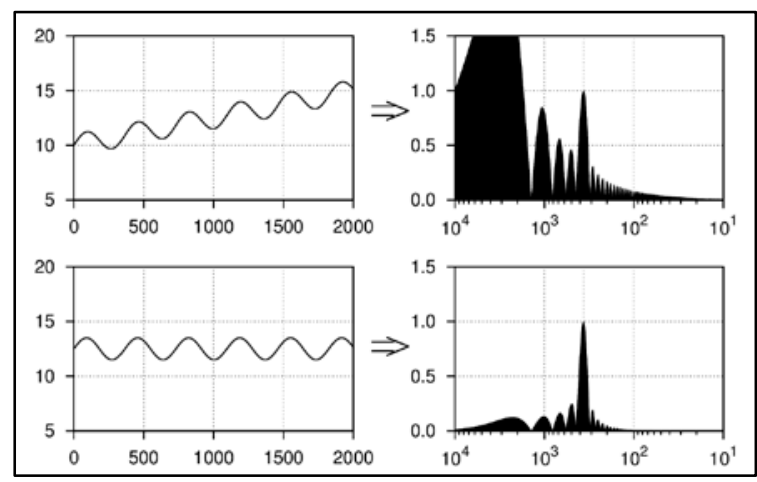

Figure 4 - Diagrammatic depiction of NDFT process on trending (upper) and non-trending (lower) timeseries.

To overcome the requirement for a uniform sample rate in the time-series a modification to Equation 1 can be applied, resulting in an implementation of a Non-uniform Discrete Fourier Transform (NDFT). This modification is shown in Equation 2.

$$
\begin{aligned}
& P(k)=\sum_{n=0}^{N-1} F_{n} \cdot e^{-i 2 \pi \frac{k}{N} n} \\
& P(\omega)=\sum_{n=0}^{N-1} F\left(t_{n}\right) \cdot e^{-i \omega t_{n}}
\end{aligned}
$$

The modification replaces the terms $2 \pi \mathrm{k} / \mathrm{N}$ with the term $\omega(=2 \pi \mathrm{h})$ allowing the power series to be specified arbitrarily over a range of frequencies ( $h$ ) and references each point's relevant time indices $\left(t_{n}\right)$. The algorithm implementing the NDFT (Equation 2) allows for calculation of the zero frequency component, $\mathrm{P}(0)$, and subsequent normalisation of the time-series to zero average magnitude. Normalisation of the signal in such way may help reduce distortion (aliasing) of the power series.

Local peak detection algorithms can be used in an iterative manner to highlight local maxima in the resulting power series with the frequency, 




Figure 5 - Comparison of local standard deviations with time across multiple temperature sensors installed in the Lasgit experiment. Vertical lineation indicates synchronicity in events or system behaviour changes.

amplitude and phase information associated with these identified peaks easily obtainable.

NDFTs can however present a positive bias in amplitude calculations due to aliasing effects (a type of distortion present in NDFTs). Additionally, frequency domain filtering (normally achieved through excluding unwanted frequency content and performing an Inverse Fourier Transform) is hampered by the arbitrary nature of the frequency domain investigated. Fourier transform procedures in general also benefit from input data with no overall trend associated with them as detailed diagrammatically in Figure 4. The peak in the lower transform is more defined due to an absence of low frequency ramping present in the conversion resulting from the trending time series (a). The frequency peak is distinguishable in both power series in Figure 4. However as the trending of the input time series increases, the low frequency ramping in the power series will obscure greater portions of the frequency domain.

\section{Non parametric data inspection techniques}

Non-parametric data inspection of a time-series consists of analysing the form of a time-series without categorising it with a mathematical relationship, i.e. not defining a continuous function to model it.
Scientific investigation of a time-series may require separation of a number of underlying contributions to the time-series. Singular Spectrum Analysis (SSA) is an analysis method capable of decomposing a time-series into a collection of mathematically independent component timeseries that sum to the original input.

The process (as described in Golyandina et al. $2001)$ involves mapping a time-series $F=\left(f_{0}, \cdots, f_{N-1}\right)$ of length $N$ into "a sequence of $[K(=N-L+1)]$ multidimensional lagged vectors" of length $L$, such that when collected into a matrix, $\mathbf{X}$, they yield:

$$
\mathbf{X}=\left[\begin{array}{ccccc}
f_{0} & f_{1} & f_{2} & \cdots & f_{K-1} \\
f_{1} & f_{2} & f_{3} & \cdots & f_{K} \\
f_{2} & f_{3} & f_{4} & \cdots & f_{K+1} \\
\vdots & \vdots & \vdots & \cdots & \vdots \\
f_{L-1} & f_{L} & f_{L+1} & \cdots & f_{N-1}
\end{array}\right]
$$

Subsequently decomposing $\mathbf{X}$ into a series of components such that $\mathbf{X}=\mathbf{X}_{1}+\cdots+\mathbf{X}_{\mathrm{L}}$. The decomposition is achieved by performing a Singular Value Decomposition (SVD) (e.g. Golyandina et al. 2001). Each component $\mathbf{X}_{\mathbf{i}}$ is associated with an eigenvalue $(\lambda)$ and eigenvector $(U)$ of the matrix $\mathbf{X X}^{\top}$ such that $\mathbf{X}_{\mathrm{i}}=\sqrt{ } \lambda_{\mathrm{i}} \mathrm{U}_{\mathrm{i}} \mathrm{V}_{\mathrm{i}}^{\top}$ and $\mathrm{V}_{\mathrm{i}}=\mathbf{X}^{\top} \mathrm{U}_{\mathrm{i}} / \mathrm{V} \lambda_{\mathrm{i}}$.

Computationally the eigenspace solution that forms the majority of the work can be achieved using the Jacobi eigenvalue algorithm (Golub and 


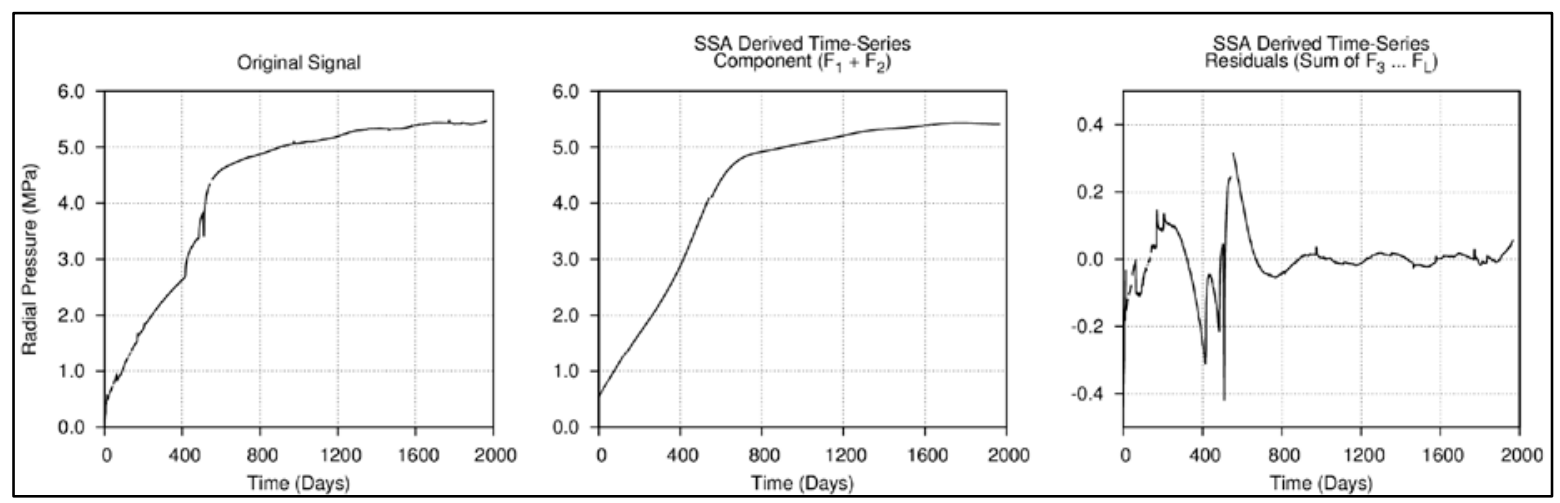

Figure 6 - Comparison of original time-series with derived component time-series and residual derived components.

van der Vorst 2000). To produce a derived timeseries component, $\mathrm{F}_{\mathrm{i}}$, from each component matrix, $\mathbf{X}_{\mathbf{i}}$, the average of the elements of the matrix along the diagonals defined by indices $i+j=$ constant, i.e. the off diagonal, is taken. The form of the derived time-series components is determined by the SSA process with no user defined specification other than number of decomposition components. Components of the input time-series typically have decreasing magnitude as the magnitude of the eigenvalue they are associated with decreases. Dependent upon form, they are typically characterised as either trends, oscillatory components or noise. Measurements of the independence of each component from the others can be performed, as can statistical tests on residual components, to confirm only noise remains. This can aid understanding of the meaning of each component or set of components.

The trends determined by SSA can also potentially be used to pre-process the original signal (e.g. be subtracted from the original signal) in order to improve frequency domain analysis or can be used to examine physical processes.

\section{Application of data analysis toolkit}

The developed toolkit has been applied to the case study (Lasgit) dataset as described earlier and an initial analysis of the dataset and assessment of the toolkit was performed.

\section{Exposed/highlighted and quantified phenomena}

Application of the spike identification algorithm to the Lasgit dataset was performed utilising a 48 hour window and a threshold of $3 \sigma_{\text {local }}$. The $3 \sigma_{\text {local }}$ threshold was chosen to coincide with the upper limit of the empirical 68-95-99.7 rule for normally distributed data. This limits false positive detection of spikes to $0.3 \%$ assuming the noise is normally distributed. The 48 hour window was chosen to ensure a large enough population from which to calculate the standard deviation at lower sample rates that occur within the Lasgit dataset. The algorithm identified 117165 spikes (approximately $0.8 \%$ of the recorded datum points), with a notable concentration of those occurring in the temperature records, and within them concentrated during the annual minimum temperature regions.

Aggregation of the local standard deviations into time-series (Figure 7) identifies second order events as indicated in Figures $3 a$ and $3 b$, with second order features in the time-series being highlighted.



Figure 7 - Second order events identified in a radial pressure record from the Lasgit dataset.

Further aggregation of local standard deviation series into groups (defined by sensor type or proximity for example) allows for a visual assessment of the independence of each timeseries. Figure 5 shows the ranked standard deviation with time of each temperature sensor installed at the Lasgit experiment. Vertical lineation indicates synchronised behaviour. The abrupt 
changes in standard deviation level indicated by the red lines in Figure 5 coincide with entries in the experimental log describing 'over-pressurisations' of the experiments fluid injection system.

Additional behaviours and events can be identified and associated in other sensor sets using this method, e.g. the reciprocating nature of the injection pumps and the closure of pressure relief holes close to the experiment.

Frequency domain analysis performed on the temperature sensors confirmed an annual temperature cycle in all down-hole positions. Phase (offset from air temperature cycle) and amplitude of the temperature cycles with depth is shown in Figure 8. The only time-series in which a daily frequency component was detected was the air temperature record of the tunnel.

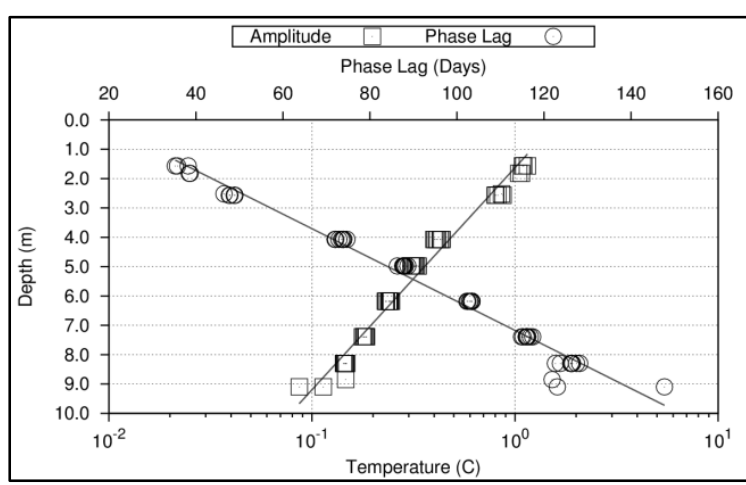

Figure 8 - Annual temperature cycle phase and amplitude information with respect to depth.

Signal component identification using SSA was performed on a reduced resolution version of the dataset to reduce the computational burden during the initial investigation phase. Representative daily values were calculated and a vector length of 365 days was applied (corresponding to an annual scale).

Figure 6 compares a radial pressure record from Lasgit with the sum of the first two components and presents the residual of the original series without the first two components. The presence of two trending components may be an indicator that two processes are driving the time-series in question.

Frequency content is observable in the residual along with anomalies that coincide with closure of the pressure relief holes near the experimental setup. The presence of this anomaly is more pronounced in other sensor types. Analysing the dataset post pressure relief hole closure may produce more informative components due to the
SSA process not attempting to account for a significant imposed change in system behaviour.

\section{Conclusions}

A toolkit has been developed to analyse long-term, large-scale datasets, particularly of the nature arising from geomechanical and geoenvironmental experiments. The toolkit has been designed to support the scientific observer, reducing the subjectivity of observations through qualitative processes.

The toolkit is capable of performing a range of analyses on non-uniform datasets including: event candidate detection; quantification and parameterisation/characterisation of time-series data; frequency domain analysis; non-parametric trend and component derivation; and system synchronisation/association visualisations.

The toolkit was applied to the Lasgit dataset as a case study and an initial analysis was undertaken. The toolkit highlighted possible events in individual time-series records and synchronisations across sensor types at specific points in time. A correlation of temperature cycles within the system (characterising both amplitude and phase) with depth was also achieved.

\section{Acknowledgements}

The research leading to these results has received funding from the European Atomic Energy Community's Seventh Framework Programme (FP7/2007-2011) under Grant Agreement No. 230357, the FORGE project.

\section{References}

Bagchi, S. and Mitra, S.K. (1999) The nonuniform discrete Fourier transform and its applications in signal processing. Kluwer Academic Publishers. ISBN: 0-7923-8281-1.

Box, G.E.P. and Jenkins, G.M. (1976) Time series analysis: forecasting and control. Revised Edition. Holden-Day.

Chatfield, C. (1989) The analysis of time series, an introduction. Fourth Edition. Chapman \& Hall.

Cuss, R.J., Harrington, J.F. and Noy, D.J. (2010) Large scale gas injection test (Lasgit) performed at the Äspö Hard Rock Laboratory: Summary report 2008. Technical report TR-10-38, British Geological Survey. 
Cuss, R.J., Harrington, J.F. and Noy, D.J., Wikman, A. and Selin, P. (2011) Large scale gas injection test (Lasgit): Results from two gas injection tests. Physics and Chemistry of the Earth, 36, 1729-1742.

Dixon, D.A., Chandler, N., Graham, J. and Gray, M.N. (2002) Two large-scale sealing tests conducted at Atomic Energy of Canada's underground research laboratory: the buffer-container experiment and the isothermal test. Canadian Geotechnical Journal, 39, No. 3, 503-518.

Golub, G.H. and van der Vorst, H.A. (2000) Eigenvalue computation in the 20th century. Journal of Computational and Applied Mathematics, 123, 35-65.

Golyandina, N., Nekrutkin, V. and Zhigljavsky, A. (2001) Analysis of Time Series Structure: SSA and Related Techniques. Chapman \& Hall/CRC.

Halpern, S. (1978) The assurance sciences, an introduction to quality control and reliability. Prentice-Hall.

O'Connor, P.D.T. (1995) Practical reliability engineering. Third Edition Revised. John Wiley \& Sons.

SKB (2006) Long-term safety for KBS-3 repositories at Forsmark and Laxemar - a first evaluation: Main Report of the SR-Can project. Technical report TR-06-09, Svensk Kärnbränslehantering $A B$.

SKB (2007) RD\&D Programme 2007 Programme for research, development and demonstration of methods for the management and disposal of nuclear waste. Technical report TR-07-12, Svensk Kärnbränslehantering $A B$. 